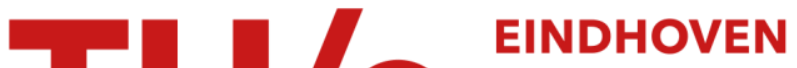 \\ UNIVERSITY OF \\ TECHNOLOGY
}

\section{Weakly nonequilibrium properties of symmetric inclusion process with open boundaries}

Citation for published version (APA):

Vafayi, K., \& Duong, M. H. (2014). Weakly nonequilibrium properties of symmetric inclusion process with open boundaries. Physical Review E - Statistical, Nonlinear, and Soft Matter Physics, 90, 052143/1-11.

https://doi.org/10.1103/PhysRevE.90.052143

DOI:

10.1103/PhysRevE.90.052143

Document status and date:

Published: 01/01/2014

\section{Document Version:}

Publisher's PDF, also known as Version of Record (includes final page, issue and volume numbers)

\section{Please check the document version of this publication:}

- A submitted manuscript is the version of the article upon submission and before peer-review. There can be important differences between the submitted version and the official published version of record. People interested in the research are advised to contact the author for the final version of the publication, or visit the $\mathrm{DOI}$ to the publisher's website.

- The final author version and the galley proof are versions of the publication after peer review.

- The final published version features the final layout of the paper including the volume, issue and page numbers.

Link to publication

\section{General rights}

Copyright and moral rights for the publications made accessible in the public portal are retained by the authors and/or other copyright owners and it is a condition of accessing publications that users recognise and abide by the legal requirements associated with these rights.

- Users may download and print one copy of any publication from the public portal for the purpose of private study or research.

- You may not further distribute the material or use it for any profit-making activity or commercial gain

- You may freely distribute the URL identifying the publication in the public portal.

If the publication is distributed under the terms of Article 25fa of the Dutch Copyright Act, indicated by the "Taverne" license above, please follow below link for the End User Agreement:

www.tue.nl/taverne

Take down policy

If you believe that this document breaches copyright please contact us at:

openaccess@tue.nl

providing details and we will investigate your claim. 


\title{
Weakly nonequilibrium properties of a symmetric inclusion process with open boundaries
}

\author{
Kiamars Vafayi* and Manh Hong Duong ${ }^{\dagger}$ \\ Department of Mathematics and Computer Science, Technische Universiteit Eindhoven, Postbus 513, 5600 MB Eindhoven, The Netherlands
}

(Received 10 June 2014; published 20 November 2014)

\begin{abstract}
We study close-to-equilibrium properties of a one-dimensional symmetric inclusion process (SIP) with finite size by coupling it to two particle reservoirs at the two boundaries with slightly different chemical potentials. The boundaries introduce irreversibility and induce a weak particle current in the system. We calculate the McLennan ensemble for the SIP, which corresponds to the entropy production, and the first-order nonequilibrium correction for the stationary state. We find that the first-order correction is a product measure and is exactly consistent with the local equilibrium measure corresponding to the steady-state density profile in the finite-size SIP, without the need to be in the thermodynamic limit. This provides a novel example for microscopic extensions of the McLennan formula and the interpretation of first-order nonequilibrium correction as entropy production.
\end{abstract}

DOI: 10.1103/PhysRevE.90.052143

PACS number(s): 02.50.Ga, 05.70.Ln

\section{INTRODUCTION}

A central concept in equilibrium statistical mechanics is the Gibbs-Boltzmann ensemble [1],

$$
\rho(\vec{\eta}) \propto e^{-\beta U(\vec{\eta})},
$$

which relates the probability $\rho(\vec{\eta})$ of finding a system in a state $\vec{\eta}$ at an inverse temperature $\beta$ to its energy function $U(\vec{\eta})$. Out of equilibrium, however, the situation is more complex and there is no simple result analogous to the Gibbs-Boltzmann distribution. There have been many attempts to provide a general formalism for nonequilibrium statistical mechanics; see, e.g., Refs. [2,3] and references therein.

Microscopic models, such as stochastic lattice gases or interacting particle systems [4,5] and interacting diffusions [6], have been found to be useful in understanding nonequilibrium phenomena. One big class of models are those of particle and heat transport, which have been used to model very diverse phenomena from phase transitions to condensation and heat and mass transport. To be able to tackle the nonequilibrium problem, instead of considering a general nonequilibrium situation, one approach is to study systems in contact to two particles or heat reservoirs at the boundaries, the so-called boundary-driven systems [7]. These, in a sense, constitute the simplest and most controllable nonequilibrium settings. In this realm, exactly solvable models play an important role, as they enable us to test various ideas and concepts about nonequilibrium [8].

A concept about nonequilibrium systems is the local equilibrium. Intuitively, it says that although on a macroscopic level the thermodynamic variables might vary significantly in space, there are regions of smaller scale which have nearly constant macro variables. Therefore, we might approximately consider such regions in equilibrium. It is important to understand the criteria and situations in which the local equilibrium holds, for example, in research related to the hydrodynamic limits of different microscopic systems [9]. This of course can be studied in two settings, for instance, in the thermodynamic limit where the size of the system becomes very large. Or, as is

\footnotetext{
*k.vafayi@tue.nl

${ }_{\dagger}^{\dagger}$ m.h.duong@tue.nl
}

our focus here, the local equilibrium can also have a meaning for finite systems in close-to-equilibrium conditions; a main question we ask is whether the first-order nonequilibrium correction to the steady state is of local equilibrium type.

In this paper we study a boundary-driven symmetric inclusion process (SIP) [10], which is a bosonic counter model to the fermionic symmetric exclusion process (SEP) [11]. The SEP and its variations have been studied extensively in the literature. The SIP, which on the other hand is new and under active research, provides an interesting model for nonequilibrium statistical mechanics where instead of hardcore-repulsion in the SEP there is attraction.

For the boundary-driven SEP and also its asymmetric version (ASEP) exact results for the stationary state were obtained via a matrix formalism [12-14]. A similar approach has not yet been successfully applied to the SIP [15], mainly due to the fact that the particle states in the SIP are unbounded in contrast to the bounded states in the SEP; i.e., at any site in the SIP there can be an arbitrary number of particles, while in the SEP the maximum occupancy is 1 . In this paper thus we follow a different strategy. We couple the system to two particle reservoirs that are nearly identical, differing by a factor $\varepsilon \ll 1$, in order to keep the system close to equilibrium. There will be, however, some nonreversibility and current flow of particles and, as a consequence, an entropy production of the order of $\varepsilon$. We studied earlier another model of interacting diffusion type [16] in related weak-coupling settings.

In the current paper, we calculate the first-order nonequilibrium correction from the reversible equilibrium state for the SIP in the context of entropy production and the McLennan ensemble. The novel feature that we observe is that the first-order nonequilibrium correction in the boundary-driven SIP with finite size matches exactly the local equilibrium (LEQ) measure. This provides a microscopic extension of the McLennan formula and correspondence with the LEQ for a classical stochastic particle system. It is worth mentioning that the local equilibrium property is usually studied for systems with very large sizes and with arbitrarily different boundaries $[17,18]$. Our focus here is, quite differently, on a finite system and boundaries that differ by a small amount of $\varepsilon$. There is, however, an important issue of proper choice for the boundaries, which is closely related to the issue of stochastic integrability that we will explain more in Sec. V. Roughly 
speaking, if one chooses the boundaries well, then one can use the LEQ measure with stationary profile exactly in place of the first-order nonequilibrium correction, which in general is much more complicated to compute.

The plan of the paper is as follows. In Sec. II, we introduce the SIP in contact with particle reservoirs and review its equilibrium properties and the corresponding thermodynamic potential. Later, in Sec. II C, we introduce the irreversible model with slightly perturbed boundary reservoirs and derive the corresponding "external" force on the system. In Sec. III, we present an elementary derivation of the stationary density profile and the corresponding local equilibrium measure, and in particular their first-order expansions. The results for reversible measure and that of stationary profile were known from Refs. [10,19], respectively. Since they are our main starting point, we review them with our choice of model parameters, for the sake of completeness. We provide a more elementary derivation of the stationary profile without explicitly using the concept of duality as in Ref. [19].

We proceed in Sec. IV to approve the McLennan proposal $[20,21]$ for the SIP that the first-order nonequilibrium correction to the equilibrium measure is the entropy production. The details of the calculation are presented in the appendix. We then confirm in Sec. IV B that a formal first-order expansion calculation in orders of $\varepsilon$ for the nonequilibrium correction to the stationary measure, as expected, yields the McLennan formula.

Comparing the entropy production and the local equilibrium measure, in Sec. IV we find that the first-order correction of the stationary measure is a product measure and corresponds exactly to the local equilibrium measure. Finally, in Sec. V, we then discuss the LEQ in the thermodynamic limit and the important issue of the choice of the boundary operators and the kind of perturbation.

\section{SYMMETRIC INCLUSION PROCESS (SIP)}

The SIP is a stochastic lattice gas introduced in Refs. [22,23] and further studied in Refs. [10,24,25]. A related model is studied in Ref. [26] from the condensation point of view. In the SIP there is an effective attraction between particles in neighboring sites. We show a state of the system by $\vec{\eta}=$ $\left(\eta_{1}, \ldots, \eta_{N}\right)$, where $\eta_{i}$ is the number of particles at the site $i$. We consider here the nearest-neighbor interactions, where a transition happen when a particle jumps to its neighboring sites, at an exponential time with a rate that depends on the occupation number of the destination site. The process in the bulk part of the system is defined via its generator corresponding to a Markov jump process, acting on the core of smooth functions $f: \mathbb{N}^{N} \rightarrow \mathbb{R}$ as observables of the system,

$$
\begin{aligned}
L_{\text {bulk }} f(\vec{\eta})= & \sum_{i} \eta_{i}\left(m+\eta_{i+1}\right)\left[f\left(\vec{\eta}^{i, i+1}\right)-f(\vec{\eta})\right] \\
& +\sum_{i} \eta_{i+1}\left(m+\eta_{i}\right)\left[f\left(\vec{\eta}^{i+1, i}\right)-f(\vec{\eta})\right] .
\end{aligned}
$$

Here $\vec{\eta}^{i, i+1}$ denotes the configuration obtained from $\vec{\eta}$ after a particle jumps from site $i$ to site $i+1$. The rate of such transition is therefore $\eta_{i}\left(m+\eta_{i+1}\right)$, in which $m$ is a parameter of the model that effectively controls the strength of diffusion

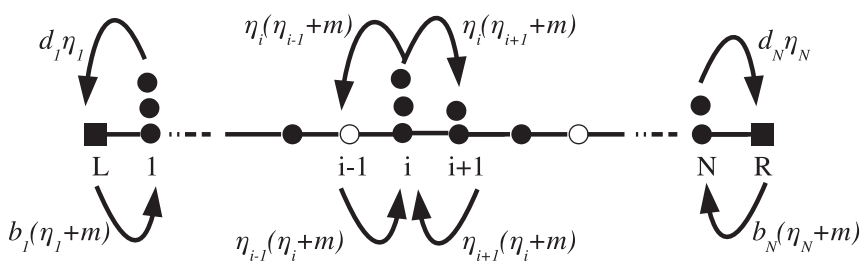

FIG. 1. A schematic representation of the symmetric inclusion process with parameter $m$. The system size is $N$, and the full circles show particles while empty circles indicate empty sites. The two squares at the boundaries with index $L$ and $R$ are the particle reservoirs. Arrows show the possible transitions and jumps of particles and the rates of such transitions are written next to the arrows. For example, a particle at site $i$ jumps to site $i+1$ with rate $\eta_{i+1}+m$ and since there are $\eta_{i}$ particles at site $i$ and the particles are indistinguishable, the total rate of such a transition is $\eta_{i}\left(\eta_{i+1}+m\right)$, which is the quantity appearing in the generator (1).

in the system. The two sums in the generator $L_{\text {bulk }}$ correspond to jumps to the right and to the left, respectively. We also consider two particle reservoirs, one at each boundary, such that particles can be put or removed at sites 1 and $N$ with rates specific of the reservoirs. The generators corresponding to the reservoirs can be written as

$$
\begin{aligned}
B_{1} f(\vec{\eta})= & b_{1}\left(m+\eta_{1}\right)\left[f\left(\vec{\eta}^{1+}\right)-f(\vec{\eta})\right] \\
& +d_{1} \eta_{1}\left[f\left(\vec{\eta}^{1-}\right)-f(\vec{\eta})\right]
\end{aligned}
$$

and

$$
\begin{aligned}
B_{N} f(\vec{\eta})= & b_{N}\left(m+\eta_{N}\right)\left[f\left(\vec{\eta}^{N+}\right)-f(\vec{\eta})\right] \\
& +d_{N} \eta_{N}\left[f\left(\vec{\eta}^{N-}\right)-f(\vec{\eta})\right],
\end{aligned}
$$

for the left and right boundaries, respectively. Here $\vec{\eta}^{i+}$ and $\vec{\eta}^{i-}$ are the configurations where a particle is added or removed at the site $i$ with rates $b_{i}$ and $d_{i}$, respectively. See Fig. 1 for an illustration of the boundary-driven SIP.

Therefore the Markovian generator for the whole system is

$$
L=L_{\text {bulk }}+B_{1}+B_{N} \text {. }
$$

In the theory of Markov processes [27, Theorem 3.16], the generator determines the time evolution of the process in the following sense:

$$
\frac{d}{d t}\left\langle f\left(\vec{\eta}_{t}\right)\right\rangle_{\vec{\eta}}=\left\langle L f\left(\vec{\eta}_{t}\right)\right\rangle_{\vec{\eta}},
$$

for any smooth function $f$. Here the symbol \langle\rangle$_{\vec{\eta}}$ indicates the average with respect to the distribution of the process started at $\vec{\eta}$ at time $t=0$. A stationary measure $v$ for the process can be defined as a measure that satisfies

$$
\int L f(\vec{\eta}) d v(\vec{\eta})=0
$$

for all functions $f$. The generator formalism is equivalent to the master equation for the evolution of the probability measure of the system,

$$
\begin{aligned}
\frac{d}{d t} v_{t}(\vec{\eta}) & =L^{*} v_{t}(\vec{\eta}) \\
& =\sum_{\vec{\eta}^{\prime}} \lambda\left(\vec{\eta}^{\prime}, \vec{\eta}\right) v_{t}\left(\vec{\eta}^{\prime}\right)-\lambda\left(\vec{\eta}, \vec{\eta}^{\prime}\right) v_{t}(\vec{\eta}),
\end{aligned}
$$


where $L^{*}$ is the adjoint generator and the transition rates $\lambda\left(\vec{\eta}^{\prime}, \vec{\eta}\right)$ can be read from the generator expression. For the states

$$
\vec{\eta}=\left(\eta_{1}, \eta_{2}, \ldots, \eta_{N}\right), \quad \vec{\eta}^{\prime}=\left(\eta_{1}^{\prime}, \eta_{2}^{\prime}, \ldots, \eta_{N}^{\prime}\right),
$$

they can be verified to be

$$
\begin{aligned}
\lambda\left(\vec{\eta}, \vec{\eta}^{\prime}\right)= & b_{1}\left(m+\eta_{1}\right) \delta_{\vec{\eta}^{1+}, \vec{\eta}^{\prime}}+d_{1} \eta_{1} \delta_{\vec{\eta}^{1-}, \vec{\eta}^{\prime}} \\
& +b_{N}\left(m+\eta_{N}\right) \delta_{\vec{\eta}^{N+}, \vec{\eta}^{\prime}}+d_{N} \eta_{N} \delta_{\vec{\eta}^{N-}, \vec{\eta}^{\prime}} \\
& +\sum_{i} \eta_{i}\left(m+\eta_{i+1}\right) \delta \vec{\eta}^{i, i+1}, \vec{\eta}^{\prime} \\
& +\eta_{i+1}\left(m+\eta_{i}\right) \delta_{\vec{\eta}^{i+1, i}, \vec{\eta}^{\prime}}
\end{aligned}
$$

An important property of the boundary-driven SIP is its self-duality property $[19,23]$, which is directly related to the issue of stochastic integrability and makes this Markov process well defined.

\section{A. Reversible stationary measure}

We first calculate the stationary measure of the SIP with closed boundaries and parameter $m$. Consider two states

$$
\begin{aligned}
\vec{\eta} & =\left(\eta_{1}, \eta_{2}, \ldots, \eta_{N}\right), \\
\vec{\eta}^{i, i+1} & =\left(\eta_{1}, \ldots, \eta_{i-1}, \eta_{i}-1, \eta_{i+1}+1, \ldots, \eta_{N}\right) .
\end{aligned}
$$

By (6), we have

$$
\begin{aligned}
& \lambda\left(\vec{\eta}, \vec{\eta}^{i, i+1}\right)=\eta_{i}\left(m+\eta_{i+1}\right), \\
& \lambda\left(\vec{\eta}^{i, i+1}, \vec{\eta}\right)=\left(\eta_{i+1}+1\right)\left(m+\eta_{i}-1\right) .
\end{aligned}
$$

As a result,

$$
\frac{\lambda\left(\vec{\eta}, \vec{\eta}^{i, i+1}\right)}{\lambda\left(\vec{\eta}^{i, i+1}, \vec{\eta}\right)}=\frac{\eta_{i}\left(m+\eta_{i+1}\right)}{\left(\eta_{i+1}+1\right)\left(m+\eta_{i}-1\right)} .
$$

The process satisfies the condition of detailed balance and has product invariant measures

$$
v(\vec{\eta})=\prod_{i=1}^{N} \gamma\left(\eta_{i}\right)
$$

where the marginals $\gamma$ can be obtained via detailed balance,

$$
v(\vec{\eta}) \lambda\left(\vec{\eta}, \vec{\eta}^{i, i+1}\right)=v\left(\vec{\eta}^{i, i+1}\right) \lambda\left(\vec{\eta}^{i, i+1}, \vec{\eta}\right) .
$$

This, together with (7), results in

$$
\begin{aligned}
& \gamma\left(\eta_{i}\right) \gamma\left(\eta_{i+1}\right) \eta_{i}\left(m+\eta_{i+1}\right) \\
& \quad=\gamma\left(\eta_{i}-1\right) \gamma\left(\eta_{i+1}+1\right)\left(\eta_{i+1}+1\right)\left(m+\eta_{i}-1\right),
\end{aligned}
$$

or, equivalently,

$$
\frac{\gamma\left(\eta_{i}\right) \eta_{i}}{\gamma\left(\eta_{i}-1\right)\left(m+\eta_{i}-1\right)}=\frac{\gamma\left(\eta_{i+1}+1\right)\left(\eta_{i+1}+1\right)}{\gamma\left(\eta_{i+1}\right)\left(m+\eta_{i+1}\right)} .
$$

Since this equation has to be valid for all values of $\eta_{i}$ and $\eta_{i+1}$, we conclude that the right-hand side and the left-hand side are both equal to a constant $\theta$. This suggests a recursive formula for $\gamma$,

$$
\gamma(n+1)=\frac{(m+n) \theta}{n+1} \gamma(n)
$$

It is convenient to write the general solution [10] in terms of the $\Gamma$ function,

$$
\gamma(n)=\frac{\theta^{n} \Gamma(m+n)}{Z_{\theta} n ! \Gamma(m)}, \quad \text { with } \quad \Gamma(s):=\int_{0}^{\infty} x^{s-1} e^{-x} d x,
$$

where $\theta$ becomes a parameter determining the average density of particles in the system and therefore can be thought of as fugacity. Here $Z_{\theta}$ is a normalization constant given by

$$
Z_{\theta}:=\sum \frac{\theta^{n} \Gamma(m+n)}{n ! \Gamma(m)}=\frac{1}{(1-\theta)^{m}} .
$$

The average number of particles at site $i$ in equilibrium can be calculated as

$$
\begin{aligned}
\rho_{i} & :=\left\langle\eta_{i}\right\rangle_{v}=\sum \eta_{i} \gamma\left(\eta_{i}\right) \\
& =\sum \frac{\eta_{i} \theta^{\eta_{i}} \Gamma\left(m+\eta_{i}\right)}{Z_{\theta} \eta_{i} ! \Gamma(m)} \\
& =\theta \frac{\partial}{\partial \theta} \log Z_{\theta} \\
& =\frac{m \theta}{1-\theta},
\end{aligned}
$$

which clearly does not depend on $i$.

The process with open boundaries also has the same reversible stationary measure. The value of $\theta$ in (10) can be obtained via considering the transitions at the two boundaries. For instance, we consider two states differing at the site 1,

$$
\vec{\eta}=\left(\eta_{1}, \ldots, \eta_{N}\right), \quad \vec{\eta}^{1+}=\left(\eta_{1}+1, \ldots, \eta_{N}\right),
$$

and with transition rates

$\lambda\left(\vec{\eta}, \vec{\eta}^{1+}\right)=b_{1}\left(m+\eta_{1}\right), \quad \lambda\left(\vec{\eta}^{1+}, \vec{\eta}\right)=d_{1}\left(\eta_{1}+1\right)$.

Similar equations hold also for the other boundary at the site $N$. Combining these with the condition of detailed balance in Eq. (9) gives that in equilibrium $\theta=\theta_{0}=\frac{b_{1}}{d_{1}}=\frac{b_{N}}{d_{N}}$. This has the interpretation that fugacity at both boundaries are equal to $\theta_{0}$. In order to simplify the formulas, we assume without loss of generality that $b_{1}=b_{N}=b, d_{1}=d_{N}=d$, and, hence, $\theta_{0}=\frac{b}{d}$, i.e., we consider two identical particle reservoirs. In equilibrium, kinetic effects (such as absolute value of $b_{i}$ and $d_{i}$ ) do not play a role, and as a consequence, choosing totally identical reservoirs at the two ends is equivalent to choosing reservoirs with only equal fugacity.

Remark 1. Note that this calculation is valid for any arbitrary number of particle reservoirs coupled to the system at different sites, in particular for only one particle reservoir. The system would need to be in contact with at least one reservoir to have the canonical reversible measure of Eq. (10). An isolated system will have the microcanonical reversible measure which is the measure (10) conditioned on having a fixed total number of particles $N_{p}$, i.e., restricted to the hyperplane $\sum \eta_{i}=N_{p}$. In the canonical measure, for the system to have a finite density of particles we need $0<\theta<1$ in Eq. (10). This corresponds to having a bigger death rate than the birth rate from the particle reservoir, i.e., $b<d$.

Remark 2. The choice of $d=b+m$ for the transition rates at boundaries is somehow special. Looking at the form of the generator for the bulk part of the system, this choice corresponds to introducing two extraboundary sites with 
indices's $0, N+1$ and freezing the number of particles at these sites to $\eta_{0}=\eta_{N+1}=b$. Particles from neighboring sites can still jump to and back from these imaginary boundary sites, as if they are annihilated or created such that the number of particles at the extraboundary site stays fixed. In this sense, that is a natural choice for the boundary rates to be made; however, the process with general $d \neq b+m$ is quite possible and well defined.

\section{B. Thermodynamic potential}

In analogy with thermodynamics, it is useful to define a thermodynamic potential $U$ such that in equilibrium

$$
v(\vec{\eta}) \propto e^{-U(\vec{\eta})},
$$

where the proportionality constant is independent of the state $\vec{\eta}$. We choose here, instead, to absorb the proportionality constant in $U$ and write an equality,

$$
U(\vec{\eta})=-\log v(\vec{\eta}) .
$$

Rewriting the detailed balance condition in terms of $U$ gives

$$
\frac{\lambda\left(\vec{\eta}, \vec{\eta}^{\prime}\right)}{\lambda\left(\vec{\eta}^{\prime}, \vec{\eta}\right)}=e^{U(\vec{\eta})-U\left(\vec{\eta}^{\prime}\right)},
$$

with the left-hand side given in Eq. (7).

Since we have product stationary measures, from (8), we conclude that $U$ is a sum of single-site potentials $V$,

$$
U(\vec{\eta})=\sum_{i=1}^{N} V\left(\eta_{i}\right)
$$

and

$$
\begin{aligned}
V(n)= & -\log \gamma(n) \\
= & -n \log \theta+m \log (1-\theta) \\
& +\log (n !)-\log \frac{\Gamma(m+n)}{\Gamma(m)} .
\end{aligned}
$$

In the bulk dynamics, the rates $\lambda\left(\vec{\eta}, \vec{\eta}^{\prime}\right)$ are non-zero only when the two states $\vec{\eta}, \vec{\eta}^{\prime}$ above differ at only two places, namely at sites $i$ and $i+1$. As a side-check, a direct calculation using $\Gamma(z+1)=z \Gamma(z)$ shows that

$$
U(\vec{\eta})-U\left(\vec{\eta}^{i, i+1}\right)=\log \frac{\eta_{i}}{m+\eta_{i}-1}-\log \frac{\eta_{i+1}+1}{m+\eta_{i+1}} .
$$

Similar calculations can be done for the boundary-driven transitions at sites 1 and $N$. Thus Eqs. (7) and (15) are satisfied.

\section{Irreversible process}

To obtain an irreversible process, we consider a small perturbation of the condition $\theta_{0}=\frac{b_{1}}{d_{1}}=\frac{b_{N}}{d_{N}}$ to achieve a system coupled to two particle reservoirs with slightly different chemical potentials. This can be done, for instance, by taking

$$
b_{1}=b+\varepsilon b, d_{1}=d ; \quad b_{N}=b-\varepsilon b, d_{N}=d .
$$

This means that we slightly perturb the system out of equilibrium by increasing the birth-rate at the left boundary while decreasing it at the right boundary. The proper choice of the boundaries and the kind of perturbation are crucial in the calculations in the rest of the paper. We discuss these issues more in Sec. V.

Following the definition in Ref. [21] of local detailed balance, which is a particular perturbation of the transition rates in the process such that

$$
\frac{\lambda\left(\vec{\eta}, \vec{\eta}^{\prime}\right)}{\lambda\left(\vec{\eta}^{\prime}, \vec{\eta}\right)}=e^{U(\vec{\eta})-U\left(\vec{\eta}^{\prime}\right)+F_{\varepsilon}\left(\vec{\eta}^{\prime}, \vec{\eta}^{\prime}\right)},
$$

we find what is equivalent of an external force, $F_{\varepsilon}\left(\vec{\eta}, \vec{\eta}^{\prime}\right)$, corresponding to the irreversible boundaries. Let us first consider these two states and their corresponding transition rates

$$
\vec{\eta}=\left(\eta_{1}, \ldots, \eta_{N}\right), \quad \vec{\eta}^{1+}=\left(\eta_{1}+1, \ldots, \eta_{N}\right),
$$

$\lambda\left(\vec{\eta}, \vec{\eta}^{1+}\right)=b_{1}\left(m+\eta_{1}\right), \quad \lambda\left(\vec{\eta}^{1+}, \vec{\eta}\right)=d_{1}\left(\eta_{1}+1\right)$,

hence,

$$
\frac{\lambda\left(\vec{\eta}^{1+} \vec{\eta}^{1+}\right)}{\lambda\left(\vec{\eta}^{1+}, \vec{\eta}\right)}=\frac{b_{1}\left(m+\eta_{1}\right)}{d_{1}\left(\eta_{1}+1\right)} .
$$

The external force $F_{\varepsilon}\left(, \vec{\eta}^{1+}\right)$ must satisfy

$$
\begin{aligned}
\frac{\lambda\left(\vec{\eta}^{1+}, \vec{\eta}^{1+}\right)}{\lambda\left(\vec{\eta}^{1+}, \vec{\eta}\right)} & =e^{U(\vec{\eta})-U\left(\vec{\eta}^{1+}\right)+F_{\varepsilon}\left(\vec{\eta}^{1+} \vec{\eta}^{1+}\right)} \\
& =\frac{\lambda_{0}\left(\vec{\eta}, \vec{\eta}^{1+}\right)}{\lambda_{0}\left(\vec{\eta}^{1+}, \vec{\eta}^{2}\right)} e^{F_{\varepsilon}\left(\vec{\eta}^{1}, \vec{\eta}^{1+}\right)} .
\end{aligned}
$$

From (21) and (22), it implies that

$$
F_{\varepsilon}\left(\vec{\eta}, \vec{\eta}^{1+}\right)=\log (1+\varepsilon) .
$$

Defining $F_{\varepsilon}\left(\vec{\eta}, \vec{\eta}^{1+}\right)=\varepsilon F_{1}\left(\vec{\eta}, \vec{\eta}^{1+}\right)+O\left(\varepsilon^{2}\right)$, it follows that

$$
F_{1}\left(\vec{\eta}, \vec{\eta}^{1+}\right)=1 .
$$

Similarly, for the other cases,

(1) $\vec{\eta}=\left(\eta_{1}, \ldots, \eta_{N}\right), \quad \vec{\eta}^{1-}=\left(\eta_{1}-1, \ldots, \eta_{N}\right)$,

$F_{1}\left(\vec{\eta}, \vec{\eta}^{1-}\right)=-1$

(2) $\vec{\eta}=\left(\eta_{1}, \ldots, \eta_{N}\right), \quad \vec{\eta}^{N+}=\left(\eta_{1}, \ldots, \eta_{N}+1\right)$,

$F_{1}\left(\vec{\eta}, \vec{\eta}^{N+}\right)=-1$

(3) $\vec{\eta}=\left(\eta_{1}, \ldots, \eta_{N}\right), \quad \vec{\eta}^{N-}=\left(\eta_{1}, \ldots, \eta_{N}-1\right)$,

$F_{1}\left(\vec{\eta}, \vec{\eta}^{N-}\right)=1$.

Remark 3. The external force $F$ is antisymmetric and nonreversible, i.e., $F\left(\vec{\eta}, \vec{\eta}^{\prime}\right)=-F\left(\vec{\eta}^{\prime}, \vec{\eta}\right)$ and satisfies the following property: For at least one set of states $\vec{\eta}_{1}, \vec{\eta}_{2}, \ldots, \vec{\eta}_{n-1}, \vec{\eta}_{n}=\vec{\eta}_{1}$

$$
\begin{aligned}
\phi_{F}\left(\vec{\eta}_{1}, \ldots, \vec{\eta}_{n}\right):= & F\left(\vec{\eta}_{1}, \vec{\eta}_{2}\right)+F\left(\vec{\eta}_{2}, \vec{\eta}_{3}\right)+\cdots \\
& +F\left(\vec{\eta}_{n-1}, \vec{\eta}_{n}\right) \neq 0 .
\end{aligned}
$$

For instance, one can show that $\phi_{F}\left(\vec{\eta}, \vec{\eta}^{1+}, \vec{\eta}^{1+, N-}, \vec{\eta}^{N-}, \vec{\eta}\right)=2 \log \left(1-\varepsilon^{2}\right) \neq 0$. Note that $G\left(\vec{\eta}, \vec{\eta}^{\prime}\right):=U(\vec{\eta})-U\left(\vec{\eta}^{\prime}\right)$ while antisymmetric, it is reversible: $\phi_{G}\left(\vec{\eta}_{1}, \ldots, \vec{\eta}_{n}\right)=0$.

\section{STATIONARY DENSITY PROFILE AND THE LOCAL EQUILIBRIUM MEASURE}

Here we consider the general nonequilibrium process with the generator (4), where the corresponding rates in particle 
reservoirs are $b_{1}, d_{1}, b_{N}$, and $d_{N}$. We define the average density of particles at the site $i$ to be

$$
\rho_{i}=\left\langle\eta_{i}\right\rangle_{\nu}
$$

where the average is taken according to the stationary measure $v$ satisfying the stationarity condition

$$
\int L f(\vec{\eta}) d v(\vec{\eta})=0
$$

for all functions $f$. Setting $f_{i}(\vec{\eta})=\eta_{i}$, a direct calculation shows that for all $2 \leqslant i \leqslant N-1$

$$
L f_{i}(\vec{\eta})=m\left(\eta_{i-1}+\eta_{i+1}-2 \eta_{i}\right),
$$

and, for the boundaries,

$$
\begin{aligned}
& L f_{1}(\vec{\eta})=b_{1} m+\left(b_{1}-d_{1}-m\right) \eta_{1}+m \eta_{2}, \\
& L f_{N}(\vec{\eta})=b_{N} m+\left(b_{N}-d_{N}-m\right) \eta_{N}+m \eta_{N-1} .
\end{aligned}
$$

These, in combination with the stationarity condition, give rise to

$$
\begin{aligned}
\rho_{i-1}+\rho_{i+1}-2 \rho_{i} & =0, \\
b_{1} m+\left(b_{1}-d_{1}-m\right) \rho_{1}+m \rho_{2} & =0, \\
b_{N} m+\left(b_{N}-d_{N}-m\right) \rho_{N}+m \rho_{N-1} & =0 .
\end{aligned}
$$

One way to solve these set of equations is to use an ansatz $\rho_{i}=\alpha+\beta i$ with two unknown parameters $\alpha$ and $\beta$. This ansatz automatically satisfies the first equation. From the other two equations we obtain

$$
\begin{aligned}
\alpha & =\frac{b_{1}\left(d_{N}-b_{N}\right) m N+\left(b_{N}+b_{1}\right) m^{2}+b_{N}\left(b_{1}-d_{1}\right) m}{\left(b_{N}-d_{N}\right)\left(b_{1}-d_{1}\right) N+\left(d_{N}+d_{1}-b_{N}-b_{1}\right) m+\left(b_{1}-d_{1}\right)\left(d_{N}-b_{N}\right)}, \\
\beta & =\frac{\left(b_{N} d_{1}-b_{1} d_{N}\right) m}{\left(b_{N}-d_{N}\right)\left(b_{1}-d_{1}\right) N+\left(d_{N}+d_{1}-b_{N}-b_{1}\right) m+\left(b_{1}-d_{1}\right)\left(d_{N}-b_{N}\right)} .
\end{aligned}
$$

Remark 4. This formula is in accordance with the result in Ref. [19]. While here we did not explicitly used the duality concept. However, duality and symmetries are the underlying reasons why such a calculation as presented here is possible, i.e., that we get a set of equations for the first moments that do not depend on the higher-order moments, which are actually more difficult to calculate.

Remark 5. Acting the generator on the function $f_{i}(\vec{\eta})=\eta_{i}$ and equating the result to the discrete gradient of the quantity $J_{i}=m\left(\eta_{i+1}-\eta_{i}\right)$ show that $J_{i}$ is the instantaneous particle current on the bond $\{i, i+1\}$ in the system. Its expectation, $J$, in the stationary state is then equal to

$$
\begin{aligned}
J:=\left\langle J_{i}\right\rangle_{v} & =m\left(\left\langle\eta_{i+1}\right\rangle_{v}-\left\langle\eta_{i}\right\rangle_{v}\right) \\
& =m\left(\rho_{i+1}-\rho_{i}\right) \\
& =m \beta .
\end{aligned}
$$

\section{A. Local-equilibrium measure}

For every general density profile $\rho_{i}$ we can associate a corresponding $\theta$ profile $\theta_{i}$, using the equilibrium relation (12)

$$
\rho_{i}=\frac{m \theta_{i}}{1-\theta_{i}},
$$

despite the fact that the equilibrium only corresponds to a constant density profile. This suggests a corresponding local-equilibrium measure (LEQ), which, similarly to the equilibrium measure, is a product measure and defined as

$$
\nu_{\mathrm{LEQ}}(\vec{\eta})=\prod_{i=1}^{N} \gamma_{\theta_{i}}\left(\eta_{i}\right),
$$

whereas in the equilibrium reversible measure (8) the marginal $\gamma_{\theta_{i}}\left(\eta_{i}\right)$ is given as

$$
\gamma_{\theta_{i}}(n)=\frac{\theta_{i}^{n} \Gamma(m+n)}{Z_{\theta_{i}} n ! \Gamma(m)} .
$$

Since there is a one-to-one correspondence between $\rho_{i}$ and $\theta_{i}$ we can freely index the local equilibrium measure by either a $\rho$ profile or a $\theta$ profile.

\section{B. Weakly nonequilibrium case}

In the weakly nonequilibrium case, with rates given as in Eq. (17), the coefficients $\alpha, \beta$ in Eq. (25) and (26) of the density profile are simplified to

$$
\begin{aligned}
& \alpha=\frac{\left(b d-b^{2}+b d \varepsilon+b^{2} \varepsilon^{2}\right) m N+2 b m^{2}+\left(b^{2}-b d+b d \varepsilon-b^{2} \varepsilon^{2}\right) m}{\left((b-d)^{2}-b^{2} \varepsilon^{2}\right) N+(2 d-2 b) m+\left(b^{2} \varepsilon^{2}-(b-d)^{2}\right)}, \\
& \beta=\frac{-2 b d m \varepsilon}{\left((b-d)^{2}-b^{2} \varepsilon^{2}\right) N+(2 d-2 b) m+\left(b^{2} \varepsilon^{2}-(b-d)^{2}\right)} .
\end{aligned}
$$


Now expanding the density $\rho_{i}$ up to the first order in $\varepsilon$ gives

$$
\rho_{i}=\alpha(\varepsilon)+\beta(\varepsilon) i+O\left(\varepsilon^{2}\right),
$$

where

$$
\begin{gathered}
\alpha(\varepsilon)=\frac{b m}{d-b}+\frac{b d m(N+1) \varepsilon}{(d-b)^{2} N+2(d-b) m-(d-b)^{2}} \\
\beta(\varepsilon)=-\frac{2 b d m \varepsilon}{(d-b)^{2} N+2(d-b) m-(d-b)^{2}} .
\end{gathered}
$$

As expected, in the case $\varepsilon=0$ we get back to the equilibrium and obtain that $\rho_{i}=\frac{b m}{d-b}=\frac{m \theta_{0}}{1-\theta_{0}}$.

\section{C. $\varepsilon$ dependence of $\theta$ and the corresponding LEQ measure}

The LEQ measure depends explicitly on $\theta$ and the relation between $\theta$ and $\rho$ is nonlinear, therefore we need to first find the appropriate expansion coefficients for $\theta$ before proceeding to do the first-order expansion for the LEQ measure. Focusing on a single-site density $\rho$ we have the corresponding $\theta$ value from (12)

$$
\theta=\frac{\rho}{m+\rho} .
$$

Now the linear expansion

$$
\rho=\rho^{(0)}+\rho^{(1)} \varepsilon+O\left(\varepsilon^{2}\right)
$$

and

$$
\theta=\theta^{(0)}+\theta^{(1)} \varepsilon+O\left(\varepsilon^{2}\right)
$$

give the following relations:

$$
\begin{aligned}
\theta^{(0)} & =\frac{\rho^{(0)}}{m+\rho^{(0)}}, \\
\theta^{(1)} & =\frac{m \rho^{(1)}}{\left(m+\rho^{(0)}\right)^{2}} .
\end{aligned}
$$

Notice that the superscripts $(0),(1)$ do not indicate the sites index, but they show the expansion order.

The LEQ measure (28) contains terms with $\theta^{n}$, which for them we have ${ }^{1}$

$$
\theta^{n}=\left(\theta^{(0)}\right)^{n}\left(1+n \varepsilon \frac{\theta^{(1)}}{\theta^{(0)}}\right)+O\left(\varepsilon^{2}\right) .
$$

Now we can express the LEQ (28) corresponding to the stationary density profile in terms of the equilibrium measure (8) up to the first order in $\varepsilon$ using (34) and (35),

$$
\begin{aligned}
v_{\mathrm{LEQ}}(\vec{\eta}) & =v_{\mathrm{EQ}}(\vec{\eta})\left(1+\varepsilon \sum_{i=1}^{N} \frac{\theta_{i}^{(1)}}{\theta_{i}^{(0)}} \eta_{i}\right)+O\left(\varepsilon^{2}\right) \\
& =v_{\mathrm{EQ}}(\vec{\eta})\left[1+\varepsilon \sum_{i=1}^{N} \frac{m \rho_{i}^{(1)}}{\rho_{i}^{(0)}\left(m+\rho_{i}^{(0)}\right)} \eta_{i}\right]+O\left(\varepsilon^{2}\right),
\end{aligned}
$$

\footnotetext{
${ }^{1}$ Neglecting the normalization constant $Z_{\theta}$, it can be shown that its contribution to the expansion is zero up to the first order in $\varepsilon$.
}

or, equivalently,

$$
\nu_{\mathrm{LEQ}}(\vec{\eta})=v_{\mathrm{EQ}}(\vec{\eta}) \exp \left[\varepsilon \sum_{i=1}^{N} \frac{m \rho_{i}^{(1)}}{\rho_{i}^{(0)}\left(m+\rho_{i}^{(0)}\right)} \eta_{i}\right]+O\left(\varepsilon^{2}\right) .
$$

From (30)-(32), we have

$$
\begin{gathered}
\rho_{i}^{(0)}=\frac{b m}{d-b}, \\
\rho_{i}^{(1)}=\frac{b d m(N+1)}{(d-b)^{2} N+2(d-b) m-(d-b)^{2}} \\
-\frac{2 b d m i}{(d-b)^{2} N+2(d-b) m-(d-b)^{2}},
\end{gathered}
$$

and therefore

$$
\nu_{\mathrm{LEQ}}(\vec{\eta})=\nu_{\mathrm{EQ}}(\vec{\eta}) \exp \left(\varepsilon \sum_{i=1}^{N} \frac{N+1-2 i}{N-1+\frac{2 m}{d-b}} \eta_{i}\right)+O\left(\varepsilon^{2}\right) .
$$

Remark 6 . In the special case that $d=b+m$ we obtain

$$
\begin{aligned}
& \rho_{i}^{(0)}=b, \\
& \rho_{i}^{(1)}=\frac{b d}{m}\left(1-\frac{2 i}{N+1}\right),
\end{aligned}
$$

and, as a result,

$$
v_{\mathrm{LEQ}}(\vec{\eta})=v_{\mathrm{EQ}}(\vec{\eta}) \exp \left[\varepsilon \sum_{i=1}^{N}\left(1-\frac{2 i}{N+1}\right) \eta_{i}\right]+O\left(\varepsilon^{2}\right) \text {. }
$$

\section{FIRST-ORDER EXPANSION: MCLENNAN FORMULA}

In the seminal work of McLennan [20], a Hamiltonian system which is weakly interacting with an environment was considered. McLennan ensembles were introduced as irreversible generalization of Gibbs ensembles for systems away but close to equilibrium. Formally, one approximates the stationary density $v$ of such system up to first order as

$$
v(\vec{\eta})=Z^{-1} \exp [-U(\vec{\eta})+\varepsilon W(\vec{\eta})]+O\left(\varepsilon^{2}\right),
$$

where $Z^{-1} \exp (-U(\vec{\eta}))$ is the equilibrium stationary measure, $W$ is the first-order non-equilibrium correction, and $\varepsilon$ is the strength of nonequilibrium forces. ${ }^{2}$ In Ref. [20] such distributions were obtained as (approximate) time-independent solutions of the modified Liouville equation. The Liouville equation was modified to incorporate the external irreversible forces. McLennan ensembles were shown to lead to linear relations between thermodynamic fluxes and the external forces, very much in the spirit of linear-response theory of Kubo [28,29].

In Refs. [21,30], the authors provided a rigorous interpretation of this formula in the context of Markov jump and diffusion processes. We now briefly recall the result in Ref. [21] for

\footnotetext{
${ }^{2}$ Note that we have set the inverse temperature $\beta=1$, since we do not study the effect of temperature here.
} 
the case of Markov jump processes. Considering a continuous Markov process on a finite state space $\Omega=\left\{\vec{\eta}, \vec{\eta}^{\prime}, \ldots\right\}$, let $\lambda\left(\vec{\eta}, \vec{\eta}^{\prime}\right)$ be the transition rate between the states $\vec{\eta} \rightarrow \vec{\eta}^{\prime}$. The probability $\mu_{t}(\vec{\eta})$ of state $\vec{\eta}$ evolves according to the master equation (5). The equilibrium distribution (indicated by subscript 0 ) fulfills the detail balanced condition

$$
\frac{\lambda_{0}\left(\vec{\eta}, \vec{\eta}^{\prime}\right)}{\lambda_{0}\left(\vec{\eta}^{\prime}, \vec{\eta}\right)}=\frac{v_{0}\left(\vec{\eta}^{\prime}\right)}{v_{0}(\vec{\eta})}
$$

where $\nu_{0}(\vec{\eta}) \propto e^{-U(\vec{\eta})}$ for some potential $U$. As mentioned in Sec. II C, in Ref. [21], the authors considered a closeto-equilibrium dynamics by replacing the detailed balanced condition by the local detailed balance condition,

$$
\frac{\lambda\left(\vec{\eta}, \vec{\eta}^{\prime}\right)}{\lambda\left(\vec{\eta}^{\prime}, \vec{\eta}\right)}=e^{F\left(\vec{\eta}, \vec{\eta}^{\prime}\right)+U(\vec{\eta})-U\left(\vec{\eta}^{\prime}\right)},
$$

where $F$ is antisymmetric and nonconservative force. To parametrize the distance to equilibrium, the authors took $F_{\varepsilon}\left(\vec{\eta}, \vec{\eta}^{\prime}\right)=\varepsilon F_{1}\left(\vec{\eta}, \vec{\eta}^{\prime}\right)$. The main result in Ref. [21] is the following asymptotic formula for the stationary distribution of the close-to-equilibrium dynamics,

$$
v_{\varepsilon}(\vec{\eta})=v_{0}(\vec{\eta}) \exp \left\{-\varepsilon \int_{0}^{\infty}\left\langle w_{1}\left(\vec{\eta}_{t}\right)\right\rangle_{\vec{\eta}}^{0} d t+O\left(\varepsilon^{2}\right)\right\},
$$

where $\langle\cdot\rangle_{\vec{\eta}}^{0}$ is the averaging over the equilibrium reference process started from $\vec{\eta}$ and

$$
w_{1}(\vec{\eta})=\sum_{\vec{\eta}^{\prime} \neq \vec{\eta}} \lambda_{0}\left(\vec{\eta}, \vec{\eta}^{\prime}\right) F_{1}\left(\vec{\eta}, \vec{\eta}^{\prime}\right)
$$

is the entropy production rate [31]. The integral in Eq. (44) is well defined depending on the process and the specific nonequilibrium perturbation, and we will address this important issue further in Sec. V. In the case of boundary-driven SIP (Sec. IV A), we are able to calculate this integral explicitly and establish its convergence.

The proof of (44) given in Ref. [21] consists of three main steps which we summarize here. The first step is to connect the nonequilibrium distribution $P_{\nu_{0}}$ on path-space with driving $F$ with the equilibrium reference distribution $P^{0}$ both starting from the equilibrium law $v_{0}$ using the Girsanov formula

$$
d P_{\nu_{0}}(\omega)=d P^{0}(\omega) e^{-A(\omega)}
$$

In this formula, $\omega=\left(\overrightarrow{\eta_{t}}\right)_{t=0}^{T}$ denotes the process trajectory in the time interval $[0, T]$, and $A$ is called the path-space action and is effectively defined by this equation. The derivation and explicit expression for the action $A$ can be found in Ref. [32, Appendix 1, Proposition 2.6]. Since the equilibrium process $P^{0}$ is time-reversal invariant, the time-antisymmetric part, $S_{\text {IRR }}^{T}$, of the action $A$ can be computed as follows:

$$
S_{\text {IRR }}^{T}(\omega):=A(\Theta \omega)-A(\omega) \stackrel{(46)}{=} \log \frac{P_{\nu_{0}}(\omega)}{P_{\nu_{0}}(\Theta \omega)},
$$

where $\Theta \omega=\left(\vec{\eta}_{T-t}\right)_{t=0}^{T}$ for any $\omega=\left(\vec{\eta}_{t}\right)_{t=0}^{T}$.

Defining $\nu_{T}^{\varepsilon}$ as the distribution of the nonequilibrium process at time $T$ starting from $v_{0}$, the second step is to express $v_{T}^{\varepsilon}$ in terms of $S_{\text {IRR }}^{T}$. We have

$$
v_{T}^{\varepsilon}(\vec{\eta})=\left\langle\delta\left(\overrightarrow{\eta_{T}}-\vec{\eta}\right)\right\rangle_{P_{v_{0}}} \stackrel{(47)}{=} v_{0}(\vec{\eta})\left\langle e^{-S_{\mathrm{IRR}}^{T}}\right\rangle_{\vec{\eta}},
$$

where the right-hand side averages over the nonequilibrium process started from the state $\vec{\eta}$. The last step is to calculate $\left\langle e^{-S_{\mathrm{IRR}}^{T}}\right\rangle_{\eta}$. For the Markov jump process, it was proven in Ref. [21] that

$$
\lim _{\varepsilon \rightarrow 0} \frac{1}{\varepsilon} \log \left\langle e^{-S_{\mathrm{IRR}}^{T}}\right\rangle_{\vec{\eta}}=-\int_{0}^{T}\left\langle w_{1}\left(\overrightarrow{\eta_{t}}\right)\right\rangle_{\vec{\eta}}^{0} d t .
$$

This, together with $v_{\varepsilon}=\lim _{T \rightarrow \infty} v_{T}^{\varepsilon}$, gives the McLennan formula (44). It is worth comparing the two formulas (43) and (44): The result in Ref. [21] provides an explanation for the McLennan formula in the sense that it identifies the correction term $W$ as the transient part of the irreversible entropy production for the process started from state $\vec{\eta}$.

\section{A. McLennan formula for the boundary-driven SIP}

We will apply the method in Ref. [21] as described above to the SIP. The small perturbation has already been introduced in Sec. II C. We now compute $w_{1}(\vec{\eta})$. By (45),

$$
\begin{aligned}
w_{1}(\vec{\eta})= & \sum_{\vec{\eta}^{\prime} \neq \vec{\eta}} \lambda_{0}\left(\vec{\eta}, \vec{\eta}^{\prime}\right) F_{1}\left(\vec{\eta}, \vec{\eta}^{\prime}\right) \\
= & \lambda_{0}\left(\vec{\eta}, \vec{\eta}^{1-}\right) F_{1}\left(\vec{\eta}, \vec{\eta}^{1-}\right)+\lambda_{0}\left(\vec{\eta}, \vec{\eta}^{1+}\right) \\
& \times F_{1}\left(\vec{\eta}, \vec{\eta}^{1+}\right)+\lambda_{0}\left(\vec{\eta}, \vec{\eta}^{N+}\right) F_{1}\left(\vec{\eta}, \vec{\eta}^{N+}\right) \\
& +\lambda_{0}\left(\vec{\eta}, \vec{\eta}^{N-}\right) F_{1}\left(\vec{\eta}, \vec{\eta}^{N-}\right) .
\end{aligned}
$$

It is worth noting that the entropy production has only boundary terms. This is because the entropy production is due to the nonequilibrium driving force $F$, and this force is only nonzero between two configurations which differ at the boundary sites 1 and $N$.

By (19) and (20), we have

$$
\begin{aligned}
\lambda_{0}\left(\vec{\eta}, \vec{\eta}^{1+}\right) & =b\left(m+\eta_{1}\right), \\
\lambda_{0}\left(\vec{\eta}, \vec{\eta}^{1-}\right) & =d \eta_{1}, \\
\lambda_{0}\left(\vec{\eta}, \vec{\eta}^{N+}\right) & =b\left(m+\eta_{N}\right), \\
\lambda_{0}\left(\vec{\eta}, \vec{\eta}^{N-}\right) & =d \eta_{N} .
\end{aligned}
$$

In Sec. II C, we already calculated

$$
\begin{aligned}
& F_{1}\left(\vec{\eta}, \vec{\eta}^{1+}\right)=F_{1}\left(\vec{\eta}, \vec{\eta}^{N-}\right)=1, \\
& F_{1}\left(\vec{\eta}, \vec{\eta}^{1-}\right)=F_{1}\left(\vec{\eta}, \vec{\eta}^{N+}\right)=-1 .
\end{aligned}
$$

Therefore,

$$
\begin{aligned}
w_{1}(\vec{\eta}) & =b\left(m+\eta_{1}\right)-d \eta_{1}-b\left(m+\eta_{N}\right)+d \eta_{N} \\
& =(b-d)\left(\eta_{1}-\eta_{N}\right) .
\end{aligned}
$$

Remark 7. We note that $\left\langle w_{1}(\vec{\eta})\right\rangle_{v}=0$, i.e., the mean rate of entropy production in equilibrium is zero. This follows from the fact that in equilibrium the average density at all sites are equal as was shown in Eq. (12).

To obtain the McLennan formula, we need to compute the time integral of $\left\langle w_{1}\left(\overrightarrow{\eta_{t}}\right)\right\rangle_{\vec{\eta}}^{0}$. In the appendix we show that

$$
\lim _{T \rightarrow \infty} \int_{0}^{T}\left\langle w_{1}\left(\vec{\eta}_{t}\right)\right\rangle_{\vec{\eta}}^{0} d t=-L^{-1} w_{1}(\vec{\eta})=-\sum_{i=1}^{N} c_{i} \eta_{i},
$$

where the coefficients $c_{i}$ given as

$$
c_{i}=A+B i \quad \text { for all } 1 \leqslant i \leqslant N,
$$


with

$$
A=\frac{N+1}{N-1-\frac{2 m}{b-d}}, \quad \text { and } \quad B=\frac{-2}{N-1-\frac{2 m}{b-d}},
$$

giving rise to the following McLennan formula for the SIP:

$$
\begin{aligned}
v_{\varepsilon}(\vec{\eta}) & =v_{0}(\vec{\eta}) \exp \left\{\varepsilon L^{-1} w_{1}(\vec{\eta})+O\left(\varepsilon^{2}\right)\right\}, \\
& =v_{0}(\vec{\eta}) \exp \left\{\varepsilon \sum_{i=1}^{N}(A+B i) \eta_{i}+O\left(\varepsilon^{2}\right)\right\} .
\end{aligned}
$$

This is identical to the local equilibrium measure corresponding to the stationary density profile that we obtained in Sec. III, i.e., the first-order nonequilibrium correction to the steady state is exactly the LEQ.

\section{B. Dyson expansion around equilibrium}

It is instructive to perform a formal perturbation expansion and see whether we recover the entropy production as in McLennan formula. We can write the generator as a sum of the reversible part and an external perturbation with strength $\varepsilon$,

$$
L=L_{0}+\varepsilon \Gamma \text {. }
$$

Expanding the (yet unknown) stationary measure $v_{\varepsilon}$ up to the first order of $\varepsilon$ in terms of the equilibrium measure $v_{0}=v_{(\varepsilon=0)}$,

$$
v_{\varepsilon}=v_{0}(1+\varepsilon h)+O\left(\varepsilon^{2}\right),
$$

which must satisfy the stationarity condition

$$
\begin{aligned}
0 & =L^{*} v_{\varepsilon}=\left(L_{0}^{*}+\varepsilon \Gamma^{*}\right)\left(v_{0}(1+\varepsilon h)+O\left(\varepsilon^{2}\right)\right) \\
& =L_{0}^{*} v_{0}+\varepsilon\left(L_{0}^{*}\left(v_{0} h\right)+\Gamma^{*} v_{0}\right)+O\left(\varepsilon^{2}\right) .
\end{aligned}
$$

This implies that

$$
L_{0}^{*}\left(v_{0} h\right)=-\Gamma^{*} v_{0},
$$

or, equivalently,

$$
h=-\frac{1}{v_{0}}\left(L_{0}^{*}\right)^{-1}\left(\Gamma^{*} v_{0}\right) .
$$

For the perturbation corresponding to the boundary rates considered in Sec. II C, we have

$$
\begin{aligned}
\Gamma f(\vec{\eta})= & b\left(m+\eta_{1}\right)\left(f\left(\vec{\eta}^{1+}\right)-f(\vec{\eta})\right) \\
& -b\left(m+\eta_{N}\right)\left(f\left(\vec{\eta}^{N+}\right)-f(\vec{\eta})\right)
\end{aligned}
$$

and

$$
\begin{aligned}
\Gamma^{*} v(\vec{\eta})= & b\left(m+\eta_{1}-1\right) v\left(\vec{\eta}^{1-}\right)-b\left(m+\eta_{1}\right) v(\vec{\eta}) \\
& -b\left(m+\eta_{N}-1\right) v\left(\vec{\eta}^{N-}\right)+b\left(m+\eta_{N}\right) v(\vec{\eta}) .
\end{aligned}
$$

The equilibrium measure has been computed in Eq. (10),

$$
\begin{aligned}
v_{0}(\vec{\eta}) & =\prod_{i=1}^{N} \gamma\left(\eta_{i}\right), \\
\gamma(n) & =\frac{\theta^{n} \Gamma(m+n)}{z_{\theta} n ! \Gamma(m)}, \\
z \Gamma(z) & =\Gamma(z+1) .
\end{aligned}
$$

We get

$$
\begin{aligned}
\Gamma^{*} v_{0}(\vec{\eta})= & b\left[\left(m+\eta_{1}-1\right) \gamma\left(\eta_{1}-1\right)\right. \\
& \left.-\left(m+\eta_{1}\right) \gamma\left(\eta_{1}\right)\right] \prod_{i=2}^{N} \gamma\left(\eta_{i}\right) \\
& +b\left[\left(m+\eta_{N}\right) \gamma\left(\eta_{N}\right)\right. \\
& \left.-\left(m+\eta_{N}-1\right) \gamma\left(\eta_{N}-1\right)\right] \prod_{i=1}^{N-1} \gamma\left(\eta_{i}\right) .
\end{aligned}
$$

By definition of $\gamma$ in Eq. (10), the first and the last terms in the right-hand side of (51) can be transformed respectively as

$$
\begin{aligned}
\left(m+\eta_{1}-1\right) \gamma\left(\eta_{1}-1\right) & =\frac{1}{\theta} \eta_{1} \gamma\left(\eta_{1}\right), \\
\left(m+\eta_{N}-1\right) \gamma\left(\eta_{N}-1\right) & =\frac{1}{\theta} \eta_{N} \gamma\left(\eta_{N}\right) .
\end{aligned}
$$

Hence it follows that

$$
\Gamma^{*} v_{0}=(b-d)\left(\eta_{N}-\eta_{1}\right) v_{0} .
$$

Substituting to (50), we obtain

$$
L_{0}^{*}\left(h v_{0}\right)=v_{0} L_{0} h=-\Gamma^{*} v_{0}=(b-d)\left(\eta_{1}-\eta_{N}\right) \nu_{0} .
$$

Therefore, finally, we obtain the first-order correction

$$
L_{0} h=(b-d)\left(\eta_{1}-\eta_{N}\right) .
$$

Comparing this with the entropy production in Eq. (49) we see that

$$
h=L_{0}^{-1} w_{1}(\vec{\eta}) .
$$

As a result, the first-order Dyson perturbation expansion becomes identical to the McLennan formula for the SIP in Sec. IV A since we have

$$
v_{\varepsilon}=v_{0}(1+\varepsilon h)+O\left(\varepsilon^{2}\right)=v_{0} \exp \left[\varepsilon h+O\left(\varepsilon^{2}\right)\right] .
$$

Remark 8 . The equality $L_{0}^{*}\left(h v_{0}\right)(\vec{\eta})=v_{0}(\vec{\eta})\left(L_{0} h\right)(\vec{\eta})$ can be verified as follows. First we define the following notation for the function $f$ and measure $v$,

$$
\langle f, v\rangle:=\int f d v .
$$

Using definition of $L_{0}^{*}$ we have that for every $f$,

$$
\left\langle f, L_{0}^{*}\left(v_{0} h\right)\right\rangle=\left\langle L_{0} f, v_{0} h\right\rangle .
$$

Furthermore, the reversibility of the process with respect to $v_{0}$ implies that

$$
\left\langle L_{0} f, v_{0} h\right\rangle=\left\langle f, v_{0} L_{0} h\right\rangle .
$$

Together, these indicate that $L_{0}^{*}\left(h v_{0}\right)=v_{0} L_{0} h$.

\section{DISCUSSIONS}

It is interesting to compare the boundary-driven SIP in this paper with the case of the boundary-driven symmetric exclusion process studied in Ref. [18] with boundaries arbitrarily far from each other, where it is shown that the Gibbs-Shannon entropy of the stationary measure converges to that of the LEQ in the thermodynamic limit as $N \rightarrow \infty$. In other words, it was shown in Ref. [18], for a rather general class of models, 
that local equilibrium is sufficient to describe the leading-order asymptotic of Gibbs-Shannon entropy. The result in the present paper is in a sense more microscopic; we study finite-size systems (fixed $N$ ) with nearly identical boundaries, and this is a different asymptotic regime, as explained in the Introduction. It is worth mentioning that the proof in Ref. [18] does not directly apply to the SIP; the SIP lacks at least one of the sufficient conditions in the proof. This is due to the fact that the particle states in the boundary-driven SIP are unbounded: Particles can be added and removed via boundaries. As a result, the entropy production rate in Eq. (49) (which is a function of the number of particles in the first and last sites) is also unbounded. Nevertheless, the integral appearing in McLennan formula (44) is well defined.

We now discuss the thermodynamic limit for the SIP. Consider coupling the SIP to two particle reservoirs that arbitrarily differ, for instance, with $\varepsilon$ not being small, and with very large $N$. Now, looking at a section of the system with size $L \ll N$, for instance, $\vec{\eta}_{i}^{(L)}=\left(\eta_{i}, \ldots, \eta_{i+L-1}\right)$, the density profile looks similar to a system of size $L$ which is coupled to two particle reservoirs whose chemical potential difference is small and of the order of $\frac{L}{N} \ll 1$. This suggests the following. Intuitively, one might expect that in the thermodynamic limit and with two boundary reservoirs with arbitrary chemical potentials, the first-order nonequilibrium correction to the stationary state is again the LEQ corresponding to the density profile. This is the property that was shown rigorously for the SIP in Ref. [17] with the help of the probabilistic technique of coupling.

An important issue in the study of close-to-equilibrium systems of the kind presented in this paper is the choice of boundaries. Good choice of the boundary operators can make the calculations feasible and other choices can make the calculations sometimes impossible. We can mention three reasons for this situation. The first reason is the so-called stochastic integrability. For example, the particular choice of boundary generators in Eqs. (2) and (3) was made in order to ensure the self-duality property of the boundary-driven SIP [23], a property that is essential for it to be exactly solvable and gives us the possibility to obtain the stationary density profile in Sec. III. There are other physically relevant choices for the boundary generators which do not have the self-duality property and thus make the calculations difficult or impossible. For example, consider the following choice for the boundary operators:

$$
B_{1} f(\vec{\eta})=b_{1}\left[f\left(\vec{\eta}^{1+}\right)-f(\vec{\eta})\right]+d_{1}\left[f\left(\vec{\eta}^{1-}\right)-f(\vec{\eta})\right]
$$

and

$$
\begin{aligned}
B_{N} f(\vec{\eta})= & b_{N}\left[f\left(\vec{\eta}^{N+}\right)-f(\vec{\eta})\right] \\
& +d_{N}\left[f\left(\vec{\eta}^{N-}\right)-f(\vec{\eta})\right],
\end{aligned}
$$

where the transition rates $b_{i}$ and $d_{i}$ do not depend on the particle configurations. This choice might be physically sensible and such process is well defined. However, the SIP with such boundaries does not have the self-duality property. The fact that we are more interest in modeling and understanding of the nonequilibrium phenomena favors choosing mathematically tractable choices.
The second reason is related to the issue of absolute continuity of the nonequilibrium perturbed process with respect to the reference equilibrium process. One process is absolutely continuous with respect to another process if the path measures of both processes are related by a density $h(\omega)$, which is called the the Radon-Nykodim derivative [32, Appendix 1, Proposition 2.6]. This absolute-continuity property is directly related to the choice of the boundary operators which induce the irreversibly in the system. It is also an essential property for the Radon-Nykodim derivative that appears in the McLennan formula to be well defined. Without this property the McLennan calculation is not possible. There are cases, e.g., as suggested in Ref. [21], that even in cases of no absolute-continuity, sometimes the perturbation expansion is still possible. This suggests that perturbation-type calculations are more generally applicable, although the resulting corrections will lack an interpretation in terms of entropy productions. The particular example provided in Ref. [21] is a general jump process where in the original unperturbed process certain jumps are forbidden but allowed in the perturbed process. The two processes are not absolutely continuous. One might consider such two systems to be partially of different physical nature. For two Markov jump processes on the same countable state space, the absolute continuity property can be established if there is no jump which is forbidden (i.e., the rate of such transition is zero) in one process but is allowed in the other. This is indeed the case with the choices of the boundaries in this paper. However, the situation is more delicate in general, and even in such cases of no absolute continuity, there is still the possibility that perturbation expansion might also fail. This can be the case if we would need to apply the inverse generator in Eq. (52) on a function that is not in this operator domain. Note that the right-hand side of this equation is related directly to the choice of the boundaries.

The third issue to consider is that for certain choices of the boundaries the system will have a linear stationary density profile, as was the case in the current paper. While this is not exactly the same as the issue of stochastic integrability, such choices for boundaries can simplify the calculations and are somehow more natural choices.

\section{ACKNOWLEDGMENT}

We thank the anonymous referees for very detailed and helpful comments. We thank Christian Maes for useful discussions. K.V. acknowledges the support of NWO VICI Grant No. 639.033.008.

\section{APPENDIX}

In this Appendix we provide some of the details of the calculation of McLennan formula in Sec. IV and then derive the McLennan formula given in Sec. IV A for the case of the SIP generator.

\section{Detailed calculation of the McLennan formula}

From the theory of continuous-time Markov processes [27, Theorem 3.16] and by definition of the generator we have 
that for all smooth functions $f$,

$$
\frac{\partial}{\partial t}\left\langle f\left(\vec{\eta}_{t}\right)\right\rangle_{\vec{\eta}}^{0}=\left\langle L f\left(\vec{\eta}_{t}\right)\right\rangle_{\vec{\eta}}^{0} .
$$

The Markov process defines a semigroup

$$
S_{t} f(\vec{\eta}):=\left\langle f\left(\vec{\eta}_{t}\right)\right\rangle_{\vec{\eta}}^{0} .
$$

By definition of the semigroup

$$
S_{t}=e^{t L},
$$

formally, it follows that

$$
\begin{aligned}
\lim _{T \rightarrow \infty} \int_{0}^{T}\left\langle f\left(\overrightarrow{\eta_{t}}\right)\right\rangle_{\vec{\eta}}^{0} d t & =\lim _{T \rightarrow \infty} \int_{0}^{T} e^{t L} f(\vec{\eta}) d t \\
& =\left.\lim _{T \rightarrow \infty} L^{-1} e^{t L} f(\vec{\eta})\right|_{t=0} ^{T} \\
& =-L^{-1} f(\vec{\eta})+L^{-1} \lim _{T \rightarrow \infty} e^{T L} f(\vec{\eta}) \\
& =-L^{-1} f(\vec{\eta})+L^{-1} \lim _{T \rightarrow \infty}\left\langle f\left(\overrightarrow{\eta_{T}}\right)\right\rangle_{\vec{\eta}}^{0} .
\end{aligned}
$$

For an irreducible Markov process, there is a unique equilibrium measure $v$ and starting from any state $\vec{\eta}$ the dynamics will converge exponentially fast to this equilibrium state. Hence $\lim _{T \rightarrow \infty}\left\langle f\left(\overrightarrow{\eta_{T}}\right)\right\rangle_{\vec{\eta}}^{0}=\langle f\rangle_{\nu}$. For computation of the McLennan formula, $f=w_{1}$ and we know that $\left\langle w_{1}\right\rangle_{v}^{0}=0$. In general we have that $L^{-1}(0)=C$, where $C$ is a constant function that does not depend on the configuration $\vec{\eta}$. As a result we can interpret $C$ as a normalization constant for the measure, which is straightforward to calculate and we omit it here. Therefore, up to an additive constant,

$$
\lim _{T \rightarrow \infty} \int_{0}^{T}\left\langle w_{1}\left(\overrightarrow{\eta_{t}}\right)\right\rangle_{\vec{\eta}}^{0} d t=-L^{-1} w_{1}(\vec{\eta})
$$

\section{Calculation of $L^{-1} w_{1}$ for SIP}

We need to calculate $\Phi(\vec{\eta})$ defined as

$$
\Phi(\vec{\eta}):=L^{-1} w_{1}(\vec{\eta}),
$$

where $L$ is the generator of the reversible process (4) with $b_{1}=b_{N}=b$ and $d_{1}=d_{N}=d$ and $w_{1}(\vec{\eta})$ given in Eq. (49). Applying both sides of the equation above with $L$ we obtain

$$
L \Phi(\vec{\eta})=w_{1}(\vec{\eta})
$$

This has the advantage that we avoid calculating $L^{-1}$ explicitly. One way to solve this equation is to use an ansatz for $\Phi(\vec{\eta})$,

$$
\Phi(\vec{\eta})=\sum_{i=1}^{N} c_{i} \eta_{i} .
$$

Now acting $L$ on $\Phi(\vec{\eta})$ gives

$$
\begin{aligned}
L \Phi(\vec{\eta})= & \sum_{i=1}^{N} c_{i} L \eta_{i} \\
= & \sum_{i=2}^{N-1} m c_{i}\left(\eta_{i-1}+\eta_{i+1}-2 \eta_{i}\right) \\
& +c_{1}\left[b m+(b-d-m) \eta_{1}+m \eta_{2}\right] \\
& +c_{N}\left[b m+(b-d-m) \eta_{N}+m \eta_{N-1}\right] .
\end{aligned}
$$

Using summation by parts to make explicit the coefficients of $\eta_{i}$, we get, after some rearrangement of the terms,

$$
\begin{aligned}
L \Phi(\vec{\eta})= & \left(c_{1}+c_{N}\right) b m+\left[c_{1}(b-d-m)+m c_{2}\right] \eta_{1} \\
& +\left[c_{N}(b-d-m)+m c_{N-1}\right] \eta_{N} \\
& +\sum_{i=2}^{N-1} m \eta_{i}\left(c_{i-1}+c_{i+1}-2 c_{i}\right) .
\end{aligned}
$$

Equating this to

$$
L \Phi(\vec{\eta})=w_{1}(\vec{\eta})=(b-d)\left(\eta_{1}-\eta_{N}\right),
$$

we will need that the coefficients of all powers of $\eta_{i}$ for all $i$ be equal on both sides of the equation. This results in

$$
c_{i-1}+c_{i+1}-2 c_{i}=0
$$

for all $2 \leqslant i \leqslant N-1$, i.e., in the bulk, and the following for the boundary conditions:

$$
\begin{aligned}
& c_{1}+c_{N}=0, \\
& c_{1}(b-d-m)+m c_{2}=b-d, \\
& c_{N}(b-d-m)+m c_{N-1}=-(b-d) .
\end{aligned}
$$

We use again a linear ansatz for $c_{i}$,

$$
c_{i}=A+B i \quad \text { for all } 1 \leqslant i \leqslant N,
$$

which will automatically satisfy the bulk discrete Laplace equations. We can find the coefficients $A$ and $B$ from the boundary equations which results in

$$
A=\frac{N+1}{N-1-\frac{2 m}{b-d}}, \quad \text { and } \quad B=\frac{-2}{N-1-\frac{2 m}{b-d}} .
$$

[1] J. W. Gibbs, Elementary Principles in Statistical Mechanics: Developed with Especial Reference to the Rational Foundation of Thermodynamics (Charles Scribner's Sons, New York, 1902).

[2] L. Bertini, A. De Sole, D. Gabrielli, G. Jona-Lasinio, and C. Landim, J. Stat. Phys. 107, 635 (2002).

[3] L. Bertini, A. De Sole, D. Gabrielli, G. Jona-Lasinio, and C. Landim, J. Stat. Phys. 135, 857 (2009).
[4] T. M. Liggett, Interacting Particle Systems, Volume 276 of Grundlehren der Mathematischen Wissenschaften [Fundamental Principles of Mathematical Sciences] (Springer-Verlag, New York, 1985).

[5] T. M. Liggett, Stochastic Interacting Systems: Contact, Voter and Exclusion Processes, Volume 324 of Grundlehren der Mathematischen Wissenschaften [Fundamental Principles of Mathematical Sciences] (Springer-Verlag, Berlin, 1999). 
[6] D. W. Stroock and S. R. Srinivasa Varadhan, Multidimensional Diffusion Processes, Volume 233 of Grundlehren der Mathematischen Wissenschaften [Fundamental Principles of Mathematical Sciences] (Springer-Verlag, Berlin, 1979).

[7] G. Eyink, J. L. Lebowitz, and H. Spohn, Comm. Math. Phys. 140, 119 (1991).

[8] G. M. Schütz, in Exactly Solvable Models for Many-Body Systems far from Equilibrium, Phase Transitions and Critical Phenomena (Academic Press, San Diego, CA, 2001), Vol. 19, pp. 1-251.

[9] S.-i. Sasa, Phys. Rev. Lett. 112, 100602 (2014).

[10] C. Giardiná, F. Redig, and K. Vafayi, J. Stat. Phys. 141, 242 (2010).

[11] F. Spitzer, Adv. Math. 5, 246 (1970).

[12] B. Derrida, E. Domany, and D. Mukamel, J. Stat. Phys. 69, 667 (1992).

[13] B. Derrida, M. R. Evans, V. Hakim, and V. Pasquier, J. Phys. A: Math. Gen. 26, 1493 (1993).

[14] B. Derrida, J. L. Lebowitz, and E. R. Speer, J. Stat. Phys. 126, 1083 (2007).

[15] Cristian Giardina, University of Modena and Reggio Emilia (private communication).

[16] F. Redig and K. Vafayi, J. Math. Phys. 52, 093303 (2011).

[17] A. Opoku and F. Redig, arXiv:1311.1620 (2013).

[18] C. Bahadoran, J. Stat. Phys. 126, 1069 (2007).

[19] G. Carinci, C. Giardinà, C. Giberti, and F. Redig, J. Stat. Phys. 152, 657 (2013).
[20] J. A. McLennan, Phys. Rev. 115, 1405 (1959).

[21] C. Maes and K. Netočný, J. Math. Phys. 51, 015219 (2010).

[22] C. Giardiná, J. Kurchan, and F. Redig, J. Math. Phys. 48, 033301 (2007).

[23] C. Giardiná, J. Kurchan, F. Redig, and K. Vafayi, J. Stat. Phys. 135, 25 (2009).

[24] S. Grosskinsky, F. Redig, and K. Vafayi, J. Stat. Phys. 142, 952 (2011).

[25] S. Grosskinsky, F. Redig, and K. Vafayi, Electron. J. Probab. 18, 23 (2013).

[26] B. Waclaw and M. R. Evans, Phys. Rev. Lett. 108, 070601 (2012).

[27] T. M. Liggett, Continuous Time Markov Processes: An Introduction, Vol. 113 Graduate Studies in Mathematics (American Mathematical Society, Providence, RI, 2010).

[28] R. Kubo, J. Phys. Soc. Jpn. 12, 570 (1957).

[29] R. Kubo, M. Yokota, and S. Nakajima, J. Phys. Soc. Jpn. 12, 1203 (1957).

[30] M. Colangeli, C. Maes, and B. Wynants, J. Phys. A: Math. Theor. 44, 095001 (2011).

[31] C. Maes, F. Redig, and A. Van Moffaert, J. Math. Phys. 41, 1528 (2000).

[32] C. Kipnis and C. Landim, Scaling Limits of Interacting Particle Systems, Vol. 320, Grundlehren der Mathematischen Wissenschaften [Fundamental Principles of Mathematical Sciences] (Springer-Verlag, Berlin, 1999). 